\title{
Diabetic gastroparesis from autonomic neuropathy: surgical considerations and changes in vagus nerve morphology
}

\author{
RJC GUY, ${ }^{*}$ JL DAWSON,† JR GARRETT, JW LAWS,§ PK THOMAS, „A AK SHARMA,ף \\ PJ WATKINS*
}

From the Diabetic Department, ${ }^{*}$ Departments of Surgery, $\dagger$ Oral Pathology and Oral Medicine, Dental School, $\ddagger$ and Diagnostic Radiology, $§$ King’s College Hospital, London, Department of Neurological Science, Royal Free Hospital, London $\|$ and the Department of Anatomy, University of Aberdeen, I UK

SUMMARY Two cases with intractable vomiting due to gastroparesis, a rare feature of diabetic autonomic neuropathy, are described. Both required surgical treatment. In the first a gastroenterostomy was complicated by reflux gastritis requiring a revision operation; in the second a gastrojejunostomy was successful. Electron microscopic studies of the vagus nerve in one of the cases showed a severe reduction in the density of unmyelinated axons, the surviving axons tending to be of small calibre. The severity of the abnormalities supports the view that diabetic gastroparesis is related to vagal denervation.

Gastroparesis is an uncommon complication of diabetes mellitus. Recurrent and debilitating vomiting from this cause is extremely rare. In exceptional cases where intractable vomiting cannot be controlled by simple measures, surgery may be needed. We describe two cases in which conservative measures failed and report the results of surgery, together with morphometric observations on the abdominal vagus nerve, obtained at the time of operation.

\section{CASE HISTORIES}

Only two patients from the Diabetic Department, King's College Hospital, have undergone elective surgery for persistent vomiting due to gastroparesis in the last ten years. Both had severe autonomic neuropathy.

\section{Case 1}

A male, born in 1947, developed insulin dependent diabetes in 1960 when aged 12 years. He had seven admissions in the next 7 years either for stabilisation or because of ketoacidosis. At the age of 22 years he had his first episode of iritis which recurred over the next two years. After 11 years of diabetes he developed peripheral neuropathy which over two years became exceptionally severe. By the time he was 25 years old he had painless

Address for reprint requests: Dr $\mathbf{R}$ Guy, Diabetic Department, King's College Hospital, Denmark Hill, London SE5 9RS, UK.

Received 6 October 1983 and in revised form 25 January 1984. Accepted 30 January 1984 ulceration of both hands and feet, bilateral Charcot $\sigma$ changes in the ankle joints, and a painless fracture of his right fifth metacarpal bone. Symptoms of an autonomic 0 neuropathy were also present. Impotent by the age of 23 , over the next six years he developed diabetic diarrhoea, symptomatic postural hypotension and retention of urine. Recurrent vomiting was, however, his most debilitating problem. It gradually progressed until he was vomiting two or three times each week by the age of 23 . Daily vomiting, often lasting twelve hours and sometimes persisting for five days was present at the age of 27 . Frequent admission in ketoacidosis followed this vomiting. In the year 19751976 , after fifteen years of diabetes, he had seven admissions in ketoacidosis. During this same period he lost weight from 72 to $60 \mathrm{~kg}$. Barium meal studies were abnormal from the age of 23 . Residual food and fluid from the previous day were present at the start of the test, barium was still present in the stomach after $23 / 4$ hours, and there was delayed passage of barium into the duodenum despite intravenous metoclopramide. When aged 28 , after 15 years of diabetes, a barium meal, recorded on videotape for later analysis, showed no effective peristaltic contractions in oesophagus or stomach, the latter emptying very slowly by gravity. The pylorus, duodenal cap and small bowel appeared normal and once barium had entered the small bowel its transit to the terminal ileum was not delayed.

Treatment with antiemetics was ineffective. Although some symptomatic benefit was initially produced by metoclopramide at night, this did not last. Combinations of metoclopramide, chlorpromazine and prochlorperazine failed to alleviate his vomiting, which could persist, despite intravenous fluids, for up to five days. Elective surgery was 
therefore undertaken for intractable symptoms in 1976 when he was aged 29 years. A gastroenterostomy was performed, anastomosing the greater curvature to the first part of the jejunum with a two finger stoma. Postoperatively he was troubled by bilious vomiting, which resulted in six admissions in the next year. Gastroscopy one year postoperatively showed a normal stoma although a considerable excess of bile was present in the stomach, refluxing from the afferent loop, with an associated gastritis. A barium meal and video again showed virtually no peristalsis of the stomach, with emptying of the barium into the jejunum via the gastroenterostomy under gravity. Because the gastroenterostomy was complicated by reflux gastritis, a revision operation was done when he was aged 30 . An antrectomy with a Roux-en-Y gastrojejunostomy and an anterior and posterior vagotomy were performed. This relieved his biliary reflux and improved his overall state. He only had two admissions with vomiting over the next five months.

Later that year, he died suddenly at home at night, aged 30 after 17 years of diabetes mellitus. Post mortem examination, full details of which have been reported previously,' showed no obvious cause of death.

\section{Case 2}

A female born in 1954 was found to be an insulindependent diabetic in 1969 when aged 15 years. By the age of 17 she had bilateral cataracts requiring lens extraction in the following year. The first episode of vomiting occurred when aged 23 and during this year she developed symptoms of postural hypotension, diabetic diarrhoea and lost both knee and ankle jerks. Over the next three years she had three admissions in ketoacidosis secondary to vomiting. Between these episodes she was asymptomatic. A gastroscopy during this time showed oesophagitis but a barium meal in 1979 was reported as normal.

In 1981 when aged 27 years, after 11 years of diabetes she became pregnant. Intractable vomiting started at the eighth week of gestation and continued over the next nine weeks despite intravenous fluids and antiemetics. The pregnancy was terminated. Monthly admission in ketoacidosis followed, secondary to vomiting with no relief from metoclopramide or pizotifen. She was then vomiting up to five times a day and food ingested over 12 hours beforehand was sometimes observed. A barium meal and video showed a fluid and food residue with poor peristalsis in oesphagus and stomach and delayed emptying.

A trial of domperidone, ${ }^{2}$ a dopamine antagonist, was started and although initially this gave some symptomatic relief with weight gain ( 54 to $59 \mathrm{~kg}$ ) over two months, there was no improvement in peristalsis as seen on video films. She then had three further admissions with ketoacidosis after vomiting in the next two months. The vomiting might last up to five days, while still on intravenous fluids. Because of continuing weight loss, (minimum weight was $49 \mathrm{~kg}$ ), severe symptoms, recurrent ketoacidosis and loss of morale, surgery was performed. A truncal vagotomy, an antrectomy and Roux-en-Y gastrojejunostomy were performed in February 1982 when she was 28 after 12 years of diabetes. A $45 \mathrm{~cm}$ retrocolic roux loop to the gastric remnant, without a valve, was made. Postoperatively she has felt better, and she has had only two admissions in seventeen months with vomiting. On neither occasion has she been ketoacidotic and has been managed on oral fluids, intramuscular metoclopramide and elevation of the head of the bed. Three weeks postoperatively, a barium meal and video showed poor peristalsis as before with negligible emptying of the stomach via the gastrojejunostomy in the supine position, but free emptying in the semierect position.

\section{Summary of case histories (see Table 1)}

Both patients were young insulin-dependent diabetics with a severe symptomatic diabetic autonomic neuropathy. Table 2 summarises their grossly abnormal autonomic function tests. In additional both had a pronounced sensorimotor neuropathy and bilateral cataracts. Neither had any evidence of nephropathy and only Case 1 had minor background retinopathy. Surgery was performed for intractable vomiting and while not wholly satisfactory in Case 1, resulted in symptomatic relief and definite improvement in gastric emptying by gravity in Case 2 .

Table 1 Summary of case histories

\begin{tabular}{|c|c|c|c|c|}
\hline \multicolumn{2}{|l|}{ Case 1} & & \multicolumn{2}{|l|}{ Case 2} \\
\hline $\begin{array}{l}\text { Duration of diabetes } \\
\text { (years) }\end{array}$ & Age (yr) & & Age (yr) & $\begin{array}{l}\text { Duration of diabetes } \\
\text { (years) }\end{array}$ \\
\hline & 12 & Diabetes mellitus & 15 & \\
\hline 10 & 23 & Bilateral cataracts & 17 & 2 \\
\hline 10 & 23 & Episodic vomiting & 23 & 7 \\
\hline 10 & 23 & Impotence & - & - \\
\hline 11 & 24 & Diabetic diarrhoea & 23 & 7 \\
\hline 12 & 25 & Charcot joints & - & - \\
\hline 13 & 26 & Background retinopathy & - & - \\
\hline 14 & 27 & Postural hypotension & 23 & 7 \\
\hline 14 & 27 & Severe vomiting & 27 & 11 \\
\hline 16 & 29 & Bladder dysfunction & - & - \\
\hline 16 & 29 & 1st operation & 28 & 12 \\
\hline 17 & 30 & 2nd operation & - & 12 \\
\hline 17 & 30 & Death & - & - \\
\hline
\end{tabular}


Table 2 Autonomic function tests

\begin{tabular}{lll}
\hline Case 1 & & Case 2 \\
\hline $120 / 85 \mathrm{~mm} \mathrm{Hg}$ & Supine blood pressure & $115 / 80 \mathrm{~mm} \mathrm{Hg}$ \\
$80 / 60 \mathrm{~mm} \mathrm{Hg}$ & Standing blood pressure & $80 / 70 \mathrm{~mm} \mathrm{Hg}$ \\
2 & Heart rate variability & $3^{*}$ \\
$102 \mathrm{bpm}$ & Resting heart rate & $110 \mathrm{bpm}$ \\
4 & Heart rate increase & $5 \dagger$ \\
& on standing & \\
\hline
\end{tabular}

${ }^{*}$ Normal over 12 . $†$ Normal increase over 15 beats/min.

\section{Abdominal vagus biopsy findings}

The anterior and posterior abdominal vagus nerves were removed at the time of gastrectomy in Case 2 . They were fixed in $3 \%$ glutaraldehyde in PIPES buffer for $3 \mathrm{~h}$ with $2 \%$ sucrose and, after washing in buffer, postfixed with $1 \%$ osmium tetroxide in buffer containing $1.5 \%$ potassium ferricyanide. ${ }^{3}$ The specimens were dehydrated in increasing concentrations of ethanol and embedded in Araldite via 1,2 epoxypropane. Ultrathin sections were stained with lead citrate and uranyl acetate and examined in an Elmiskop 101 electron microscope. The quantitative morphometric studies were performed on nonoverlapping photographs at a magnification of $\times 17000$. The total area examined was $3000 \mu \mathrm{m}^{2}$. The findings were compared with the control values obtained by Sharma and Thomas ${ }^{4}$ from patients

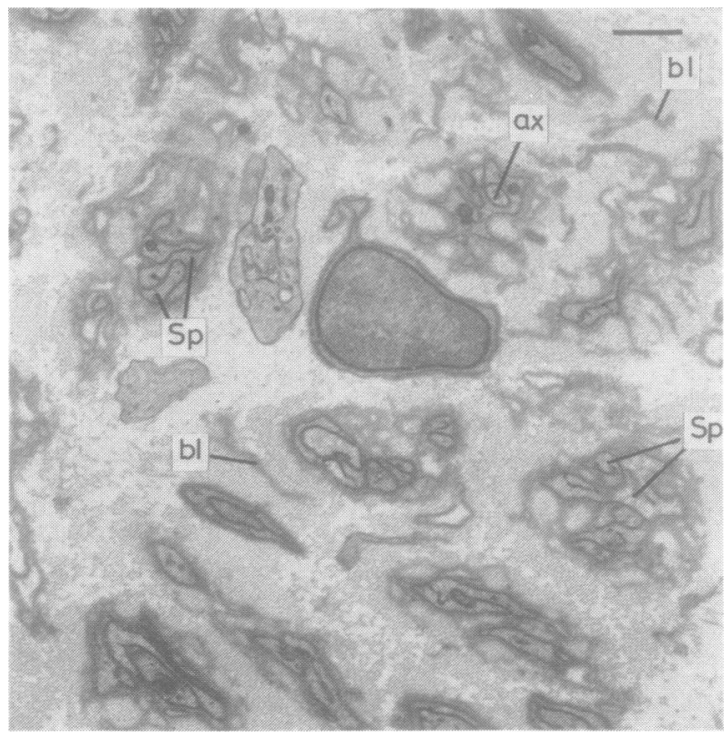

Fig 1 Electron micrograph of transverse section through abdominal vagus nerve from Case 2, showing considerable loss in density of unmyelinated axons (ax). Groups of Schwann cell processes (Sp) are present, unassociated with axons, and portions of basal lamina (bl) are seen, unassociated with Schwann cell processes. Bar $=1 \mu \mathrm{m}$.

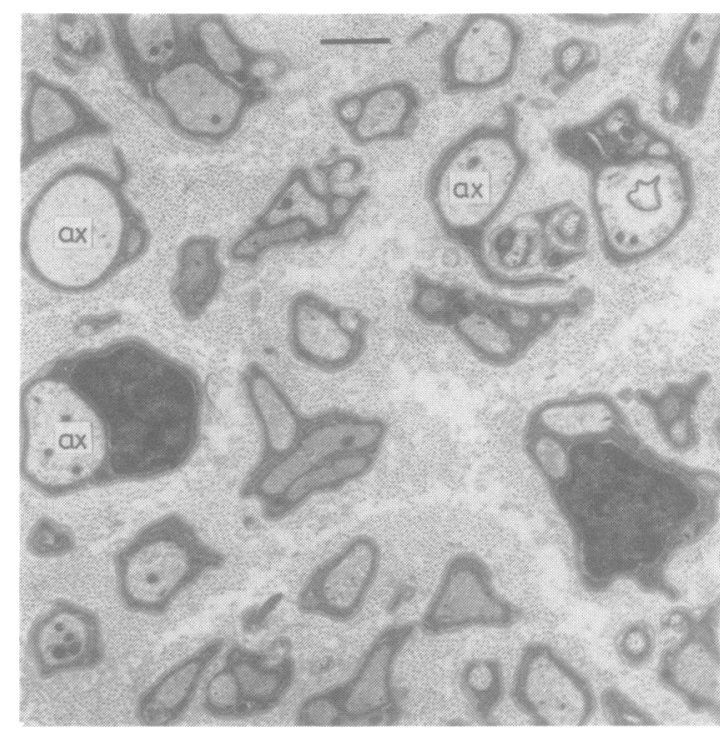

Fig 2 Electron micrograph of transverse section through abdominal vagus nerve of a control subject aged 24, showing a dense population of unmyelinated axons (ax) and associated Schwann cell processes. Bar $=1 \mu \mathrm{m}$.

undergoing vagotomy for the treatment of peptic ulceration. This nerve normally contains very few myelinated axons.

The specimens from the patient showed a severe reduction in the density of unmyelinated axons. This was $49000 / \mathrm{mm}^{2}$ as compared with the control value of $209000 \pm 66 / \mathrm{mm}^{2}$ (mean $\pm \mathrm{SD}$ ). Those that remained were generally of small diameter. Representative areas from the patient and a control subject aged 24 years are shown in figs 1 and 2 . Numerous Schwann cell subunits unassociated with axons were present in the patient. The basal lamina surrounding the Schwann cells was thickened in the nerve from the patient. This was assessed quantitatively. Its thickness was measured at 5-16 equidistant points around the perimeter of 30 Schwann cell subunits, the number of measurements depending upon the size of the subunit. Only regions in true transverse section were assessed, tangentially-cut areas being avoided. A value of $293 \cdot 5 \pm 12 \mathrm{~nm}$ (mean $\pm \mathrm{SE}$ ) was obtained. Similar observations on a control subject aged 24 yielded a value of $72 \cdot 5 \pm$ $11 \mathrm{~nm}$. This difference is significant $(\mathrm{p}<0 \cdot 01)$. Case 2 also showed multiple portions of basal lamina unassociated with Schwann cell processes (fig 1). Not infrequently they enclosed dense collections of collagen fibrils, the appearance resembling "collagen pockets" from which the surrounding Schwann cell processes had disappeared (fig 3 ). The amount of endoneurial collagen was increased. The 


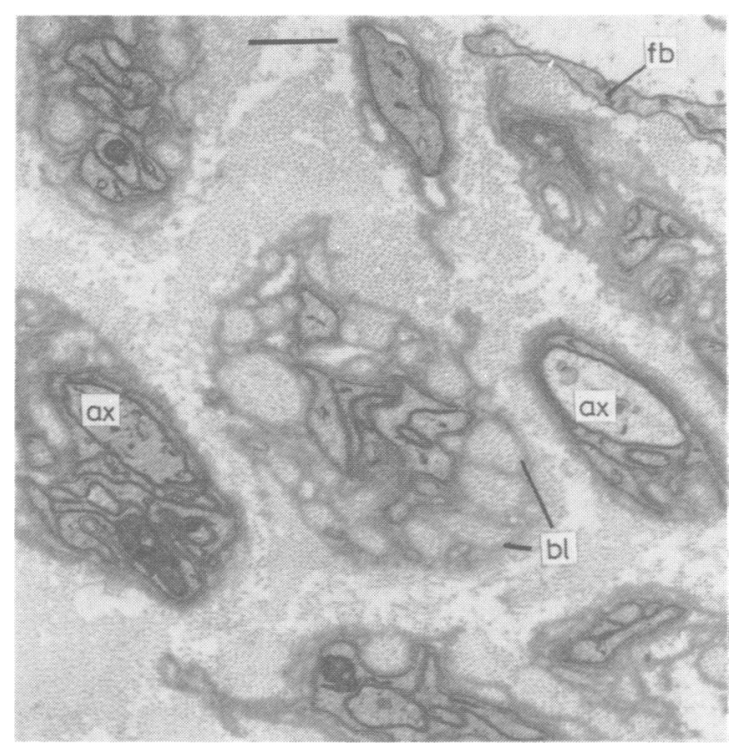

Fig 3 Electron micrograph of transverse section through abdominal vagus of Case 2 showing portions of Schwann cell basal lamina (bl) enclosing pockets of collagen fibrils. $\mathrm{ax}$, axons; fb, fibroblast process. Bar $=1 \mu \mathrm{m}$.

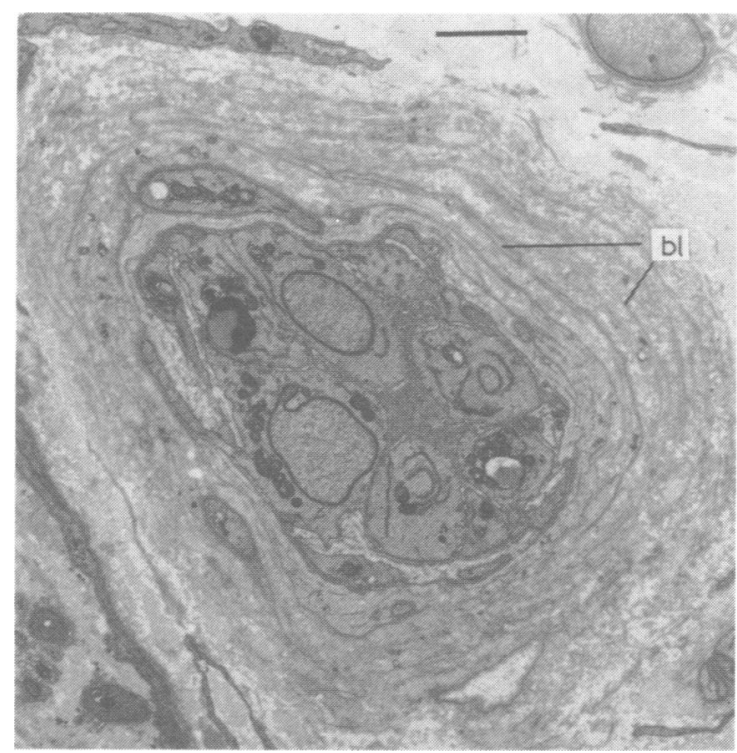

Fig 4 Electron micrograph of endoneurial capillary from abdominal vagus nerve of Case 2, surrounded by thickened zone composed of reduplicated basal lamina (bl) and associated collagen fibrils. Bar $=2 \mu \mathrm{m}$.

endoneurial capillaries were abnormal, being surrounded by a thick zone composed of reduplicated basal laminae with associated collagen fibrils (fig 4).

\section{Discussion}

Gastric retention with delayed stomach emptying was described over 40 years ago $^{5}$ and linked with diabetic autonomic nerve dysfunction by Rundles in $1943 .^{6}$ The term gastroparesis diabeticorum was used by Kassander to describe asymptomatic gastric retention.?

Gastroparesis is a feature of autonomic neuropathy ${ }^{6-10}$ but it is an uncommon complication. $^{8}$ Vomiting is generally intermittent, while debilitating symptoms from intractable vomiting are rare. Mild nonspecific symptoms such as nausea, epigastric discomfort, anorexia, bloated feelings, and borborygmi have been rather dubiously attributed to gastroparesis. Delayed emptying, ${ }^{7}$ residual food and absent or ineffective peristalsis ${ }^{9}$ may be discovered at barium meal examination. A Crosby capsule often fails to pass through the duodenum because of poor peristalsis.

Gastric vagal denervation has long been considered to cause gastroparesis, although surgical denervation causes abnormalities of gastric motility which are not identical to those in diabetic neuropathy. The present study demonstrates for the first time that there are severe structural abnormalities in the vagus nerve (from Case 2), supporting the view that vagal denervation may be responsible for gastroparesis. Gastric smooth muscle abnormalities have also been observed in both these cases, and consist of very striking hyaline bodies of unknown origin interspersed amongst the smooth muscle cells. They were also demonstrated in smooth muscle from other organs. "'

Observations on the pathological changes in diabetic gastroenteropathy have not so far been extensive. Smith ${ }^{12}$ found abnormalities in the innervation of the oesophagus in 18 out of 20 unselected cases of diabetes. None had clinical dysphagia or neuropathy. Kristensson et al ${ }^{13}$ reported loss of myelinated fibres in the vagus nerve of three necropsied cases of diabetic neuropathy, one of whom had diabetic diarrhoea and radiologically demonstrated gastric atony. Duchen et al, ${ }^{1}$ reported necropsy findings in five insulin-dependent diabetics with sensorimotor and autonomic neuropathy. Severe loss of myelinated fibres was found in the vagus nerves. A conspicuous feature was the presence of inflammatory changes in the autonomic ganglia and in and around bundles of axons in the walls of viscera, but not in nerve trunks.

The abdominal vagus in Case 2 from the present study showed a severe loss of unmyelinated axons. The surviving axons tended to be of small calibre. This suggests either a preferential loss of axons of larger size or a diffuse involvement with the pres- 
ence of small regenerating axons. These findings support the idea that diabetic gastroparesis is related to vagal denervation.

The substantial thickening of the Schwann cell basal lamina is of interest. It has been commented upon by Bischoff ${ }^{14}$ in sural nerve biopsies from patients with diabetic neuropathy, although it is not a regular feature. The vascular changes observed in the present case, are also not consistently present in diabetic neuropathy. No inflammatory infiltration was observed.

Assessment and treatment of gastroparesis is very difficult. While isotopic scanning tests for emptying of solids may be normal, ${ }^{15}$ tests for emptying of liquids and solids together, ${ }^{16}$ may be abnormal. Also, since the symptoms are intermittent, subjective improvement sometimes occurs despite persistently abnormal radiographs. ${ }^{9}$

Various treatments have been suggested including gastric suction and a six-meal-a-day diet, ${ }^{7}$ ambenonium chloride,${ }^{8}$ bethanechol and a right lateral decubitus position, ${ }^{9}$ metoclopramide ${ }^{16}$ and more recently domperidone. ${ }^{2}$ Symptomatic improvement which can be achieved with metoclopramide does not correlate closely with an improvement of radionuclide measurement of gastric emptying. ${ }^{17}$ Manometric recordings to assess gastric motor function have shown an increase in gastric motility with intravenous metoclopramide ${ }^{18}$ although others have only shown an improvement after subcutaneous bethanechol. ${ }^{19}$

The cases described here were of exceptional severity. Few patients have symptoms that necessitate surgery. Wooten ${ }^{9}$ described two such cases; one had a pyloroplasty and the other a Billroth type 1 gastrectomy. He concluded that a gastrojejunostomy would have been more appropriate, but advised against operating. Brownlee ${ }^{20}$ reported one case who underwent a pyloroplasty. After operation symptoms persisted and a barium meal continued to show delayed gastric emptying. Some improvement occurred with metoclopramide. Our two cases show that severe vomiting, recurrent hospital admissions in ketoacidosis and weight loss may necessitate an operation. To avoid biliary reflux and to facilitate drainage of the gastric remnant, a Roux-en-Y gastrojejunostomy was recommended. A vagotomy and antrectomy was performed to minimise the chance of any stomal ulceration, although with the degree of vagal degeneration, this may be unnecessary. Even postoperatively, gastric stasis may be such that adequate emptying will occur only in the semierect or erect position.

The results of surgery in our two cases and those reported by others are not always satisfactory and even gastrojejunostomy does not always alleviate the problem. Nonetheless, when intractable vomiting from gastroparesis does occur, surgery may be the only appropriate treatment.

We thank Dr DA Pyke for his helpful advice and encouragement, and Dr RHM King and Miss JM Workman for collaboration in the electron microscope studies. RJCG is supported by a research grant from Pfizer Ltd.

\section{References}

' Duchen LW, Anjorin A, Watkins PJ, Mackay JD. Pathology of autonomic neuropathy in diabetes mellitus. Ann Int Med 1980;92:301-3.

${ }^{2}$ Gordon JJ, Joseph RE. Domperidone in patients with postprandial upper gastrointestinal disease. In: Progress with Domperidone, a Gastrokinetic and Antiemetic Agent. Roy Soc Med Int Cong Symp Ser 1981;36:67-75.

${ }^{3}$ Langford LA, Coggeshall RE. The use of potassium ferricyanide in neural fixation. Anat Rec 1980;197:297303.

${ }^{4}$ Sharma AK, Thomas PK. Quantitative studies on age changes in unmyelinated nerve fibres in the vagus nerve in man. In: Kunze $K$ and Desmedt JE, eds. Studies in Neuromuscular Diseases. Basel: Karger, 1975:211-20.

5 Lepore MJ, Golden R. Syndrome due to deficiency of vitamin B complex. JAMA 1941;117:918-23.

- Rundles RW. Diabetic neuropathy. Medicine (Baltimore) $1945 ; 24: 111-60$.

${ }^{7}$ Kassander P. Asymptomatic gastric retention in diabetes (gastroparesis diabeticorum). Ann Intern Med 1958; 48:797-812.

${ }^{8}$ Zitomer BR, Gramm HF, Kozak GP. Gastric neuropathy in diabetes mellitus: clinical and radiological observation. Metabolism 1968;17:199-211.

9 Wooten RL, Meriwether TW. Diabetic gastric atony: a clinical study. JAMA 1961;176:1082-7.

${ }^{10}$ Hodges FJ, Rundles RW, Hanelin J. Roentgenologic study of the small intestine. Dysfunction associated with neurologic disease. Radiology 1947;49:659-73.

11 Duchen LW. Neuropathology of the autonomic nervous system in diabetes. In: Bannister $R$, ed. Autonomic Failure. A Textbook of Clinical Disorders of the Autonomic Nervous System. Oxford University Press, 1982:437-52.

${ }_{12}$ Smith B. Neuropathology of the oesophagus in diabetes mellitus. J Neurol Neurosurg Psychiatry 1974; 37:1151-4.

${ }^{13}$ Kristensson K, Nordborg C, Olsson Y, Sourander P. Changes in the vagus nerve in diabetes mellitus. Acta Pathol Microbiol Scand (A) 1971;79:684-5.

${ }^{14}$ Bischoff A. Diabetische Neuropathie: pathologische Anatomie, Pathophysiologie und Pathogenese aufgrund elektronen-mikroskopischer Untersuchungen. Dtsch Med Wochenschr 1968;93:237-41.

15 Scarpello JHB, Barber DC, Hague RV, et al. Gastric emptying of solid meals in diabetes. $\mathrm{Br}$ Med $\mathrm{J}$ 1976; 2:671-3. 
${ }^{16}$ Campbell IW, Heading RC, Tothill P, et al. Gastric emptying in diabetic autonomic neuropathy. Gut 1977; 18:462-7.

17 Snape WJ, Battle WM, Schwartz SS, et al. Metoclopramide to treat gastroparesis due to diabetes mellitus. Ann Intern Med 1982;96:444-6.

${ }^{18}$ Malagelada JR, Rees WD, Mazzott LJ, et al. Gastric motor abnormalities in diabetic and postvagotomy gastroparesis: effect of metoclopramide and bethanechol. Gastroenterology 1980;78:286-93.

19 Fox S, Bemar J. Pathogenesis of diabetic gastroparesis: a pharmacologic study. Gastroenterology 1980;78:757-63.

${ }^{20}$ Brownlee M, Kroopf SS. Metoclopramide for gastroparesis diabeticorum. $N$ Engl $J$ Med 1974; 291: $1256-8$. 